\title{
IMPLEMENTAÇÃO ESTRATÉGICA E ESTILO DO EXECUTIVO: UM ESTUDO SOBRE ALINHAMENTO ESTRATÉGICO E DESEMPENHO EM PEQUENAS E MÉDIAS INDÚSTRIAS DO RIO GRANDE DO NORTE
}

\section{RESUMO}

As pequenas e médias empresas sofrem uma série de restrições no mercado onde competem, sejam provocados pela escassez de recursos ou por dificuldades de inserção. Para que a empresa se desenvolva bem no mercado onde atua, destaca-se o papel do executivo no processo estratégico. A presente pesquisa teve como objetivo geral verificar a existência de alinhamento entre estilo de executivo e as estratégias e o impacto deste no desempenho das pequenas e médias indústrias de Mossoró/RN. Metodologicamente, trata-se de uma pesquisa descritiva com coleta de dados por levantamento: foi elaborado um questionário estruturado e autoadministrado, adaptado do modelo de Håkonsson et al (2012). Foram feitas questões fechadas, utilizando escala likert de 5 pontos. As hipóteses do modelo foram testadas pelo teste de Mann-Whitney. Constatou-se que a grande maioria das empresas apresenta um desalinhamento entre os estilos dos executivos e a estratégia da empresa, sendo o principal desalinhamento do tipo: estilo gerente - estratégia analítica. A hipótese geral, de que o alinhamento entre o estilo cognitivo e a estratégia da empresa leva a um melhor desempenho foi corroborada. Os resultados da pesquisa corroboram os resultados obtidos por Håkonsson et al (2012).

Palavras-chave: Implementação Estratégica; Estilos Cognitivos do Executivo; Pequenas e Médias Empresas.

\section{STRATEGY IMPLEMENTATION AND THE EXECUTIVE STYLE : A STUDY ON STRATEGIC} ALIGNMENT AND PERFORMANCE IN SMALL AND MEDIUM INDUSTRIES OF RIO GRANDE DO NORTE

\begin{abstract}
Small and midsize businesses are a number of restrictions on the market where they compete, they are caused by lack of resources or integration difficulties . For the company to develop and market in which it operates, highlights the role of the executive in the strategic process. This research investigation aims check for alignment between executive style and strategies and the impact of the performance of small and medium industries Mossley / RN . Methodologically, it is a descriptive study with data collection by surveying a structured and self-administered questionnaire , adapted from Håkonsson et al (2012 ) model was developed . Closed questions were asked, using Likert 5-point scale . The assumptions of the model were tested by Mann- Whitney test. It was found that the vast majority of companies presents a misalignment between the styles of business and enterprise strategy, the main type of misalignment : Style manager analytical strategy. The general hypothesis, that the alignment between cognitive style and enterprise strategy leads to improved performance has been confirmed. The results corroborate the results obtained by Håkonsson et al (2012).
\end{abstract}

Keywords: Strategic Implementation; Cognitive Styles Executive; Small and Medium Enterprises. 
Implementação Estratégica e Estilo do Executivo: Um Estudo sobre Alinhamento Estratégico e Desempenho em Pequenas e Médias Indústrias do Rio Grande do Norte

\section{IMPLEMENTACIÓN DE LA ESTRATEGIA Y EL ESTILO EJECUTIVO : UN ESTUDIO SOBRE LA ALINEACIÓN ESTRATÉGICA Y EL DESEMPEÑO DE LAS PEQUEÑAS Y MEDIANAS INDUSTRIAS DE RÍO GRANDE DO NORTE}

\section{RESUMEN}

Las pequeñas y medianas empresas son una serie de restricciones en el mercado donde compiten, son causados por la falta de recursos o dificultades de integración. Para la empresa para desarrollar y comercializar en el que opera , destaca el papel del ejecutivo en el proceso estratégico. Esta investigación tiene como objetivo la investigación para comprobar la alineación entre el estilo y las estrategias de poder ejecutivo y el impacto de la evolución de las pequeñas y medianas industrias Mossley / RN . Metodológicamente, se trata de un estudio descriptivo, con la recogida de datos mediante encuestas a un cuestionario estructurado y auto-administrado, adaptado de Håkonsson et al modelo ( 2012 ) fue desarrollado. Se hicieron preguntas cerradas, utilizando la escala de Likert de 5 puntos . Los supuestos del modelo se analizaron mediante la prueba de Mann- Whitney. Se encontró que la gran mayoría de las empresas presenta un desajuste entre los estilos de negocio y estrategia de la empresa, el principal tipo de desalineación : manager Style estrategia analítica. La hipótesis general, que la alineación entre el estilo cognitivo y la estrategia de la empresa conduce a un mejor desempeño ha sido confirmada. Los resultados corroboran los resultados obtenidos por Håkonsson et al (2012).

Palabras clave: Implementación Estratégica; Estilos Cognitivos Executivo; Pequenas y Medianas Empresas.

Alvaro Fabiano Pereira de Macedo ${ }^{1}$ Liana Holanda Nobre ${ }^{2}$ Jansen Maia Del Corso ${ }^{3}$ Alceu Souza ${ }^{4}$

\footnotetext{
${ }^{1}$ Mestre em Ciências Contábeis pela Universidade de Brasília - UnB. Professor da Universidade Federal Rural do Semi-Árido - UFERSA. Brasil. E-mail: alvarofabiano@ ufersa.edu.br

${ }^{2}$ Mestre em Administração pela Universidade Estadual do Ceará - UECE. Professor da Universidade Federal Rural do Semi-Árido - UFERSA. Brasil. E-mail: liana.nobre@ufersa.edu.br

${ }^{3}$ Doutor em Management Science pelo Esade Escuela Superior de Administración y Dirección de Empresas da Univers, Espanha. Professor da Pontifícia Universidade Católica do Paraná - PUC/PR. Brasil. E-mail: del.corso@pucpr.br

${ }^{4}$ Doutor em Administração de Empresas pela Fundação Getulio Vargas - FVG. Professor da Pontifícia Universidade Católica do Paraná - PUC/PR. Brasil. E-mail: alceu.souza@pucpr.br
} 


\section{INTRODUÇÃO}

O interesse nas pequenas e médias empresas (PMEs) se justifica pela importância que as mesmas têm na economia mundial, representando uma parcela significativa do PIB dos países desenvolvidos e em desenvolvimento. As PMEs têm se revelado importantes para as economias nacionais, e ganharam maior importância em um país onde existe grande concentração de renda e exclusão social como o Brasil. Estas organizações contribuem, ainda, com a geração de empregos e o fortalecimento de economias locais, pois têm a capacidade de gerar postos de trabalho a um custo bem menor do que nas grandes.

Para Audretsch (apud NAJBERG; PUGA; OLIVEIRA, 2000), há duas visões contraditórias sobre as PMEs: se por um lado, elas impõem custos excessivos para a economia como resultado de escalas de produção ineficientes, que implicam baixa produtividade e baixos salários para os seus trabalhadores; em outra perspectiva, estas unidades nascentes são agentes de mudança, com um papel crucial na inovação tecnológica. Além disso, é através desses estabelecimentos que milhões de trabalhadores chegam ao mercado de trabalho.

Para além do tamanho, há outras características igualmente relevantes que diferenciam as PMEs e grandes empresas, tais como a racionalidade limitada do gestor, a baixa diversificação de produtos e serviços, e a sobreposição entre propriedade e gestão (NOBRE, 2005). Tais características das PMEs se mostram relevantes ao interferirem na gestão das pequenas e médias empresas, que se torna cada vez mais dependente da habilidade individual do proprietário-gestor (ROCHA, 2001).

Para Cooper (1981), os diferentes ambientes internos implicam em caminhos também diferentes no que concerne à formulação e implementação da estratégia. O contexto no qual cada estratégia é gerenciada também varia de acordo com o grau de desenvolvimento da organização. Ao caracterizar o ambiente onde atuam as pequenas empresas, Lima (2003) ressalta ainda que este é evolutivo, dinâmico e caracterizado pelas mudanças contínuas, e que seus membros devem promover a coevolução de modo a resguardar a condição de operação das empresas em compatibilidade com as características do meio ambiente. Ramos (2005) reitera que a elaboração de estratégias em pequenas empresas é um processo muito mais complexo do que a simples escolha racional de seus atores, indicando assim que a elaboração da estratégia reflete a intensidade com que as pressões para a similaridade são percebidas e interpretadas pelo seu estrategista. Desta forma, o papel atribuído ao agente responsável pela implementação da estratégia cresce se reveste de grande importância, sendo considerado inclusive como elemento chave da implementação da estratégia.

Partindo deste contexto, Håkonsson et al (2012) desenvolveram um modelo sobre o alinhamento estratégico entre o estilo cognitivo do executivo e a implementação estratégica. Os autores partiram da hipótese de que o alinhamento entre determinados estilos cognitivos de executivo e as estratégias implementadas levam a um melhor desempenho da empresa. Com base nesse modelo proposto, esta pesquisa pretende resolver a seguinte problemática: o estilo cognitivo do executivo da PMEs alinhado à estratégia implementada afeta o desempenho das pequenas e médias indústrias de transformação de Mossoró/RN?

Esse estudo tem como objetivo geral verificar a existência de alinhamento entre estilo de executivo e as estratégias das pequenas e médias indústrias de transformação de Mossoró/RN, e tem como objetivos específicos: (1) identificar os estilos cognitivos dos executivos das pequenas e médias indústrias de transformação de Mossoró; (2) diagnosticar as estratégias implementadas por tais gestores, e (3) avaliar o impacto do alinhamento proposto no modelo de Håkonsson et al (2012) sobre o desempenho destas empresas.

Buscando atingir esses objetivos, o presente artigo está estruturado da seguinte forma: a introdução apresenta o tema, justificativa e objetivos da pesquisa; a fundamentação teórico-empírica traz definições dos estilos de executivo e de implementação estratégica, como elementos de análise; procedimentos metodológicos; descrição e análise dos dados; considerações finais e recomendações.

\section{FUNDAMENTAÇÃO TEÓRICO-EMPÍRICA}

O conceito de Estratégia é tema de profunda discussão no meio acadêmico que gera debates quer seja no âmbito conceitual, quer seja na perspectiva de aplicação. Essa multiplicidade de visões sobre a estratégia, apesar de não formar um consenso, indica que existem orientações interessantes e construtivas; sem, contudo, oferecer respostas definitivas neste campo (GIMENEZ; RAMOS; FERREIRA, 2005; MINTZBERG; QUINN, 2001). Håkonsson et al (2012) consideram que os executivos das pequenas empresas, independentemente do tipo de propriedade ou de negócios, implementam uma estratégia baseada no seu estilo cognitivo. Julga-se relevante o entendimento de que os conceitos associados ao tema de análise implementação de estratégia em pequenas e médias empresas, assim como o estilo cognitivo do gestor possam assegurar que os resultados obtidos colaborem à luz do conhecimento da área. 


\subsection{Estilos Cognitivos do Executivo - Aspectos Conceituais e Diagnóstico}

As características dos executivos e gestores há muito tem sido objeto de estudo no campo da Administração. Especificamente no que se refere às pequenas e médias empresas, tais estudos se justificam uma vez que os dirigentes dessas organizações geralmente imprimem sua marca pessoal, agindo como um filtro para a formulação de ações e decisão estratégica (SANTOS et al, 2007). A forma como o gestor influencia a estratégia tem sido estudada especificamente a partir de duas abordagens (FERREIRA; RAMOS, 2004): a abordagem das características individuais, que se refere a traços comuns a estes executivos, tais como propensão ao risco, lócus interno de controle, entre outros (KORUNKA et. al., 2003); e a abordagem cognitiva, que se refere aos processos de pensamento e padrões empregados pelos gestores para processar a informação na tomada de decisão.

Imerso na abordagem cognitiva, Håkonsson et. al. (2012) defiunem os estilos dos executivos a partir de duas dimensões de análise: os níveis de delegação e a maneira como estes lidam com a incerteza. A dimensão "nível de delegação" se refere à forma pela qual as decisões são tomadas na organização: em um extremo inferior, encontra-se um baixo grau de delegação, indicando que as decisões são tomadas exclusivamente pelo executivo, sem consulta ou participação de outros membros da gestão; no outro extremo, encontra-se o alto grau de delegação, que conta com o envolvimento dos demais gestores da organização.

A dimensão "aversão à incerteza" se refere à maneira pela qual os executivos lidam com a incerteza em relação ao futuro e como estes incorporam esta reação às suas atividades. Assim, no extremo inferior desta dimensão, estariam os executivos que tem baixa predisposição para evitar a incerteza, o que se traduz em um maior foco no longo prazo, ações mais arriscadas, preferência pela motivação ao invés de controle; enquanto no extremo superior, os executivos tem preferência pelas decisões de curto prazo, tem maior foco no controle e preferem informações agregadas (HÅKONSSON et. al., 2012). Esta dimensão é equivalente à teoria da adaptação-inovação, desenvolvida por Kirton, em 1976 (KIRTON, BAYLEY, GLENDINNING, 1991),que propôs um continuum com as maneiras preferidas pelos indivíduos para a resolução de problemas e tomada de decisão: em um extremo, o autor identificou o comportamento adaptador, caracterizado pela ordem, precisão, preocupação com detalhes, conformidade, disciplina e eficiência; e no outro extremo, o autor identificou o estilo inovador, que engloba os indivíduos com que preferem solucionar problemas através de alternativas arriscadas, apresentam uma forte tendência pela busca de novidades e pela exploração de novos horizontes.

A partir destas dimensões, Håkonsson et. al. (2012) identificaram quatro tipos básicos de executivos: Maestro, Gerente, Produtor e Líder.Os executivos identificados como Maestro apresentam baixas pontuações para ambas as dimensões de análise: nível de delegação e aversão à incerteza. Eles preferem se envolver diretamente nas tomadas de decisões, e preferem agir no longo prazo. Os executivos diagnosticados na categoria Gerente tem alta pontuação na dimensão evitar a incerteza, e baixa pontuação na dimensão nível de delegação. Estes são caracterizados por manter foco nas decisões de curto prazo e apresentam preferência por atividades de controle e alto nível de detalhe das informações. A categoria Líder é caracterizada por uma pontuação baixa na dimensão aversão à incerteza, e uma pontuação alta na dimensão nível de delegação. Estes executivos são reconhecidos por promoverem o debate para a tomada de decisão, tem uma preferência pelas decisões de longo prazo, e geralmente tem um estilo proativo de gestão. Por fim, na categoria Produtores estão enquadrados os executivos que apresentam alto nível de delegação e baixa propensão ao risco. Håkonsson et. al. (2012) sugerem que tais gestores, embora não tenham a iniciativa de inovar por eles mesmos, sejam susceptíveis a permitir que outros gestores tomem medidas arriscadas enquanto ele mantém o foco global e o controle da situação.

\subsection{Implementação da Estratégia - Aspectos Conceituais e Diagnóstico}

Porter (1981) compreende a estratégia como uma maneira de posicionar a empresa no ambiente onde compete. Hitt, Ireland e Hoskinsson (2008) concebem a estratégia de maneira similar à de Porter: para os autores, a estratégia é um conjunto coordenado de ações definido para explorar competência e obter vantagem competitiva. A concepção de Porter de estratégia vislumbra as estratégias deliberadas. Já Mintzberg classifica a estratégia em dois grupos: a estratégia deliberada, aquela que foi planejada e implementada de forma organizada numa ótica de dentro para fora; e a estratégia emergente onde a lógica funcional se inverte, tendo como pressuposto a aprendizagem organizacional, onde as ações tomadas agora servem de feedback para as ações seguintes, ao passo que esse ciclo vicioso (ou virtuoso) configure-se numa estratégia (MINTZBERG; WATERS, 1996). Para Mintzberg, portanto, a implementação e a formulação da estratégia não estão intrinsecamente separados. 

em Pequenas e Médias Indústrias do Rio Grande do Norte

Esta visão, no entanto, não é consensual. Andrews (1996) conceitua a estratégia como um processo organizacional inseparável de sua estrutura, comportamento e cultura, do qual se pode extrair dois importantes aspectos: formulação e implementação estratégica.

Independente da discussão sobre a separação temporal entre formulação e implementação da estratégia, Andrews (1996) avança no estudo sobre a implementação da estratégia. Para o autor, ela é composta de várias subatividades, que requerem dos gestores uma liderança que é fundamental no sucesso da implementação. Ele destaca que o sucesso de uma estratégia em uma organização não é passível de ser conseguido por outras empresas, mesmo que estas seguissem o mesmo caminho. Assim, a característica mais importante para uma estratégia é a sua unicidade, representada pela criativa combinação de recursos e oportunidades.

O processo da implementação de uma estratégia implica em transformar a mesma em resultados; isso significa colocar frente a frente decisões estratégicas e fatores organizacionais como estrutura, coordenação, incentivos e informações inseridos num contexto organizacional com dimensões referentes à gestão da mudança, cultura organizacional, estrutura de poder e liderança.(CAETANI et al 2011). Dessa forma o grande desafio de gerenciar a execução da estratégia é gerenciar as mudanças das organizações. Vários estudos tem sido desenvolvidos no que concerne aos problemas e dificuldades que afetam a implementação da estratégia nas empresas e como mais recorrente tem-se o fluxo de informações, à coordenação interfuncional e o comportamento corporativo vinculado à liderança (KAPLAN; NORTON, 1996; HREBINIAK, 2006).

Dentre as inúmeras abordagens para se categorizar a estratégia da empresa, destaca-se a taxonomia desenvolvida por Miles e Snow, que tem sido exaustivamente testada e comprovada (Ghoshal et. al., 2003; Hambrick, 2003). Para Miles e Snow (1978), as empresas desenvolvem padrões de comportamento estratégico relativamente estáveis na busca de bom alinhamento com as condições ambientais percebidas pela administração. $\mathrm{O}$ enquadramento da estratégia da empresa em uma das categorias estabelecidas pelos autores depende da forma como os executivos percebem as condições ambientais e tomam decisões sobre estruturas e processos para interagir com esse ambiente percebido. Assim, o modelo proposto por Miles e Snow (1978) identifica quatro posturas estratégicas: Prospectora, Analítica, Defensiva e Reativa. Para Gimenez et al(1999), as duas categorias mais contrastantes são a prospectora e a defensiva: enquanto a primeira se caracteriza por uma busca e geração constante de inovação, a segunda se caracteriza por uma restrição de seus domínios de mercado e maximização da eficiência. Os autores pontuam ainda que a categoria analítica é uma composição das duas estratégias anteriores, que busca maximizar a eficiência em uma atividade central e estável da empresa, mas mantém um componente dinâmico, oriundo da estratégia prospectora. A estratégia reativa, por sua vez, é considerada por muitos autores como uma forma ineficaz de comportamento estratégico, pois não carrega nenhum aspecto inovador; os ajustes são forçados pelo ambiente externo. (GIMENEZ et al, 1999).

No modelo proposto por Håkonsson et. al. (2012), o enquadramento da estratégia da empresa em uma das categorias do modelo de Miles e Snow se dá através da análise de duas dimensões: exploração e aproveitamento. Em um extremo, a dimensão exploração é compreendida como a busca pelo novo e engloba o desenvolvimento de novos produtos e/ou processos e a procura de novos mercados; já o aproveitamento busca a maximização da eficiência dos processos e mercados já existentes. Para os autores, a estratégia Defensiva enfoca baixa eficiência na exploração e alta eficiência no aproveitamento. A estratégia Analítica tem alta pontuação nas duas dimensões de análise, é muito complexa e requer no processamento das informações uma gama elevada de conhecimento, sendo indicada para empresas que optam por atuar nos dois enfoques (JANSEN et. al., 2008). A estratégia Prospectora apresenta baixa pontuação no aproveitamento e alta pontuação na exploração, e requer uma análise contínua do ambiente externo, pois lida com um escopo de informações amplo. E a estratégia Reativa, por sua vez, apresenta baixa pontuação tanto no item exploração quanto no item aproveitamento. A figura 1 apresenta o modelo de Håkonsson et. al. (2012), no que diz respeito ao alinhamento entre estilos cognitivos e estratégia. 
Implementação Estratégica e Estilo do Executivo: Um Estudo sobre Alinhamento Estratégico e Desempenho em Pequenas e Médias Indústrias do Rio Grande do Norte

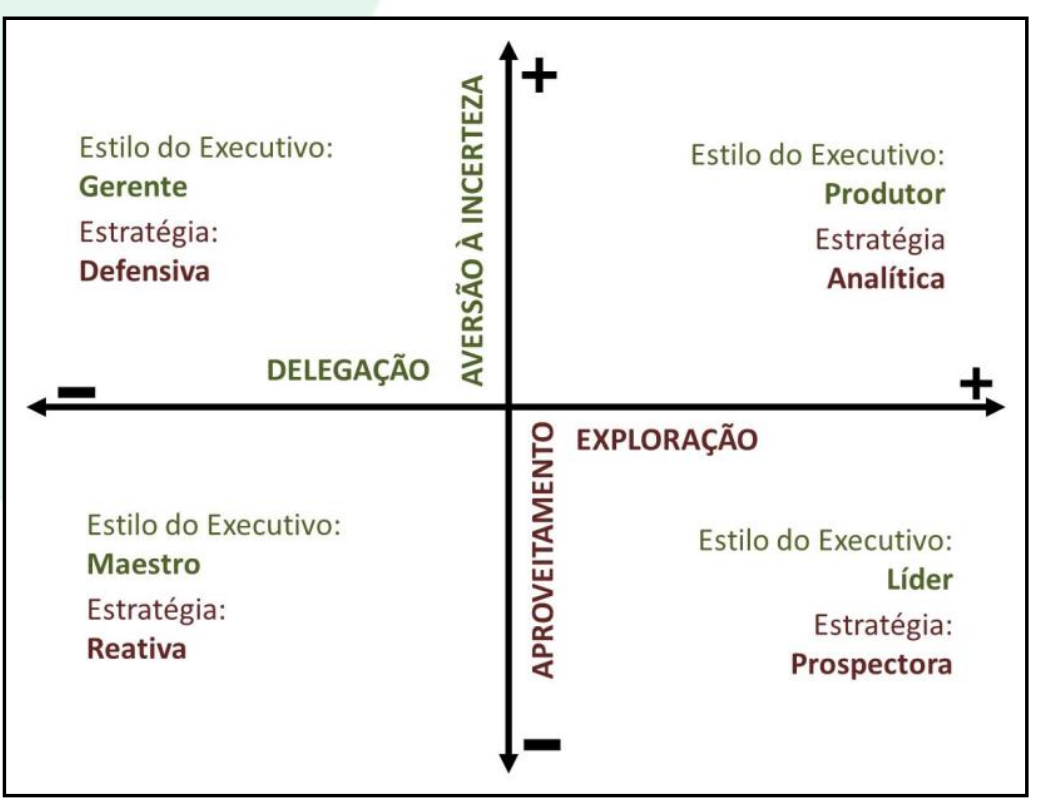

Figura 1 - Alinhando estilo cognitivo do executivo com a estratégia.

Fonte: adaptado de Håkonsson et al (2012)

A forma como a estratégia é implementada depende em grande parte do porte da empresa: nas médias e grandes empresas, esse processo é estabelecido pela alta gerência, que repassa aos demais setores. No caso das pequenas empresas o processo é idealizado e implementado pelo gestor que acumula várias funções e desempenha inúmeras tarefas simultaneamente. Håkonsson et. al. (2012) esclarecem que nessas empresas onde ocorre falta de recursos humanos e financeiros, dificuldades de tecnologia, baixa produtividade, o sucesso da estratégia é vital na maioria dos casos para a própria sobrevivência da empresa, daí a importância do alinhamento entre o estilo do executivo da empresa e a estratégia adotada.

\section{PROCEDIMENTOS METODOLÓGICOS}

Com o propósito de analisar o alinhamento entre os estilos de executivo, as estratégias e o desempenho da empresa, foi realizada uma pesquisa do tipo descritivo, cujo objetivo é a identificação e análise dos fenômenos estudados (GIL, 2002).Utilizando-se o levantamento como procedimento metodológico, foi elaborado um questionário estruturado e autoadministrado. $\mathrm{O}$ questionário foi adaptado do modelo de Håkonsson et. al. (2012), sendo dividido em quatro seções: a primeira seção procurava diagnosticar o estilo cognitivo do executivo, através de questões que operacionalizavam os comportamentos referentes ao nível de delegação e à aversão à incerteza; a segunda seção correspondia ao diagnóstico da estratégia, no que se refere às questões relacionadas às dimensões de exploração e aproveitamento. Na terceira seção, foi indagado ao respondente sobre o desempenho da empresa em relação aos seus concorrentes; e, por fim, na última seção, estavam as questões relacionadas ao gênero, idade e escolaridade do respondente e tempo de existência da empresa. Foram feitas questões fechadas, utilizando escala likert de 5 pontos.

As hipóteses testadas neste trabalho são aquelas propostas pelo modelo de Håkonsson et. al. (2012):

- Hipótese Geral: Maior alinhamento entre o estilo cognitivo do executivo e a estratégia leva a um melhor desempenho da empresa.

- Hipótese 1: Maior alinhamento entre o estilo Maestro e uma estratégia Reativa leva a um melhor desempenho.

- Hipótese 2: Maior alinhamento entre um estilo Gerente e uma estratégia Defensiva leva a um melhor desempenho.

- Hipótese 3: Maior alinhamento entre o estilo Líder e uma estratégia Prospectora leva a um melhor desempenho.

- Hipótese 4: Maior alinhamento entre o estilo Produtor e uma estratégia Analítica leva a um melhor desempenho.

As hipóteses combinam o estilo executivo com a estratégia implementada. Cada hipótese indica os efeitos da interação com o alinhamento entre estilo executivo e a estratégia. $\mathrm{O}$ quadro 1 apresenta resumidamente os motivos pelos quais se espera um menor desempenho das empresas no caso de desalinhamento entre os estilos cognitivos dos executivos e as estratégias das empresas. 

em Pequenas e Médias Indústrias do Rio Grande do Norte

\begin{tabular}{|c|c|c|c|c|}
\hline & MAESTRO & GERENTE & LÍDER & PRODUTOR \\
\hline Reativa & $\begin{array}{c}\text { Desempenho superior } \\
\text { esperado }\end{array}$ & $\begin{array}{l}\text { O foco no controle e } \\
\text { na eficiência não se } \\
\text { ajusta à baixa } \\
\text { necessidade } \\
\text { melhoramento de } \\
\text { processos, típica das } \\
\text { estratégias reativa e } \\
\text { prospectiva. }(*)\end{array}$ & $\begin{array}{l}\text { O líder requer a } \\
\text { exploração } \\
\text { oportunidades de } \\
\text { mercado, o que não } \\
\text { se ajusta à estratégia } \\
\text { reativa. }\end{array}$ & $(*)$ \\
\hline Defensiva & $\begin{array}{l}\text { O maestro foca muito } \\
\text { pouco em questões de } \\
\text { eficiência e controle, } \\
\text { enquanto } \\
\text { estratégias defensiva } \\
\text { e analítica requerem } \\
\text { tal característica para } \\
\text { o contínuo } \\
\text { melhoramento dos }\end{array}$ & $\begin{array}{c}\text { Desempenho superior } \\
\text { esperado }\end{array}$ & $\begin{array}{l}\text { O líder foca muito } \\
\text { pouco em questões de } \\
\text { eficiência e controle, } \\
\text { enquanto } \\
\text { estratégias defensiva } \\
\text { e analítica requerem } \\
\text { tal característica para } \\
\text { o contínuo } \\
\text { melhoramento dos } \\
\text { seus processos. }(* *)\end{array}$ & $\begin{array}{l}\text { O produtor delega } \\
\text { para diminuir sua } \\
\text { necessidade de } \\
\text { processar informação } \\
\text { e, então, facilitar a } \\
\text { inovação e promover } \\
\text { o debate, faltando o } \\
\text { foco necessário para } \\
\text { a estratégia } \\
\text { defensiva. }\end{array}$ \\
\hline Prospectiva & $\begin{array}{lr}\text { seus processos. } & \text { Por } \\
\text { outro lado, } & \text { as }\end{array}$ & $\begin{array}{l}\text { As estratégias } \\
\text { prospectiva e }\end{array}$ & $\begin{array}{l}\text { Desempenho superior } \\
\text { esperado }\end{array}$ & $(*)$ \\
\hline Analítica & $\begin{array}{l}\text { estratégias } \\
\text { prospectiva } \\
\text { analítica } \\
\text { maior grequerem } \\
\text { delegação } \text { e este } \\
\text { estilo se caracteriza } \\
\text { por ser centralizador. }\end{array}$ & \begin{tabular}{lr} 
analítica & \multicolumn{2}{r}{ requerem } \\
maior grau de & para \\
delegação & \\
melhor explorar as \\
oportunidades \\
mercado, \\
principalmente na \\
inovação de produtos.
\end{tabular} & $(* *)$ & $\begin{array}{c}\text { Desempenho superior } \\
\text { esperado }\end{array}$ \\
\hline
\end{tabular}

Quadro 1- Estilo cognitivo do executivo x estratégia implementada: desempenho esperado das empresas alinhadas e dos diferentes tipos de desalinhamento.

Fonte: Elaborado pelos autores (2012)

Quanto à técnica de análise dos dados, foi aplicado o teste de Mann-Whitney para testar as hipóteses propostas. O teste de Mann-Whitney é aplicado para testar se duas amostras independentes foram extraídas de populações com médias iguais quando os dados não são normalmente distribuídos e/ou quando a amostra é pequena (FÁVERO et. al., 2009). Assim, a hipótese nula é que não há diferença estatisticamente significante entre as médias das amostras a serem testadas. Os autores ressaltam que este é um dos testes não-paramétricos mais utilizados e mais poderosos. Neste trabalho, o teste U MannWhitney foi realizado a partir do software SPSS, que possibilitou a análise dos dados, elaboração de tabelas e gráficos auxiliares.

\section{DESCRIÇÃO E ANÁLISE DOS DADOS}

A presente pesquisa foi realizada no período de junho e julho de 2012, com 35 (trinta e cinco) executivos de pequenas e médias empresas da indústria transformadora da cidade de Mossoró/RN. As empresas pesquisadas fazem parte da base de dados da Federação das Indústrias do Rio Grande do Norte -
FIERN, e foram excluídas da população as indústrias extrativistas, as empresas com menos de 10 funcionários, as empresas que não tinham dados atualizados para contato. A seguir, são descritos o perfil dos respondentes, a caracterização das empresas objetos da pesquisa e os resultados das técnicas de análise utilizadas no estudo.

\subsection{Perfil do Respondente e da Empresa}

O estudo mostrou uma significativa representação masculina entre os empresários na amostra analisada. Foram identificados $71,4 \%$ de respondentes do sexo masculino e $28,6 \%$ do sexo feminino. A média de idade dos pesquisados foi de 39,5 anos, sendo que $45,4 \%$ dos indivíduos pesquisados têm entre 36 e 46 anos. Tais números sugerem maturidade e experiência dos executivos da amostra, bem como a escolaridade predominante de pelo menos ensino superior em andamento $(68,6 \%)$, caracterizando executivos com maior grau de conhecimento formal. O detalhamento do grau de escolaridade é apresentado na Tabela 1. 
Implementação Estratégica e Estilo do Executivo: Um Estudo sobre Alinhamento Estratégico e Desempenho em Pequenas e Médias Indústrias do Rio Grande do Norte

Tabela 1 - Nível de escolaridade dos respondentes

\begin{tabular}{|l|c|}
\hline \multicolumn{1}{|c|}{ ESCOLARIDADE } & PERCENTUAL \\
\hline Não estudou / Ensino fundamental incompleto & 2,9 \\
\hline Ensino fundamental completo & 8,6 \\
\hline Ensino médio incompleto & 11,4 \\
\hline Ensino médio completo & 8,6 \\
\hline Ensino superior incompleto & 40,0 \\
\hline Ensino superior completo & 11,4 \\
\hline Pós-graduação completa & 17,1 \\
\hline Total & $100 \%$ \\
\hline
\end{tabular}

Fonte: Elaborado pelos autores (2012)

Quanto à empresa, a média de tempo de funcionamento é de 16,6 anos, sendo que 48,8\% das empresas funcionam entre 8 e 18 anos. Diante do fato que mais de $50 \%$ das empresas se extinguem antes de completar cinco anos (DONATO, 2011), pode-se inferir que as empresas estudadas têm uma longevidade alta comparada às pequenas e médias empresas brasileiras.

\subsection{Diagnósticos do Estilo do Executivo e da Estratégia de Negócio}

O diagnóstico do estilo cognitivo dos executivos e da estratégia de negócios foi realizado a partir do conjunto de respostas dadas pelos próprios respondentes. A partir da operacionalização de variáveis que compunham as dimensões "nível de delegação" e "aversão à incerteza", a grande maioria dos executivos das organizações estudadas se enquadrou no estilo Gerente, totalizando $68,6 \%$, seguido por Maestro, com 25,7\%, e Produtor, com $5,7 \%$. Nenhum dos executivos foi identificado na categoria Líder. $\mathrm{O}$ estilo cognitivo detectado na pesquisa é coerente com as características dos gestores das pequenas e médias empresas elencadas por Nobre (2005): tais executivos teriam um comportamento centralizador, conservador, pouco agressivo e pouco afeito a riscos.

No que se refere às estratégias diagnosticadas, a partir do agrupamento das variáveis operacionais, $65,7 \%$ dos respondentes categorizaram a estratégia da empresa como Analítica; 28,6\% dos entrevistados categorizaram a estratégia como Defensiva; 5,7\% dos pesquisados categorizaram a estratégia da empresa como Reativa; enquanto nenhum dos executivos pesquisados identificou como estratégia Prospectora.

Os estilos cognitivos dos executivos e as estratégias diagnosticadas no presente estudo estão representados graficamente na Figura 2. 


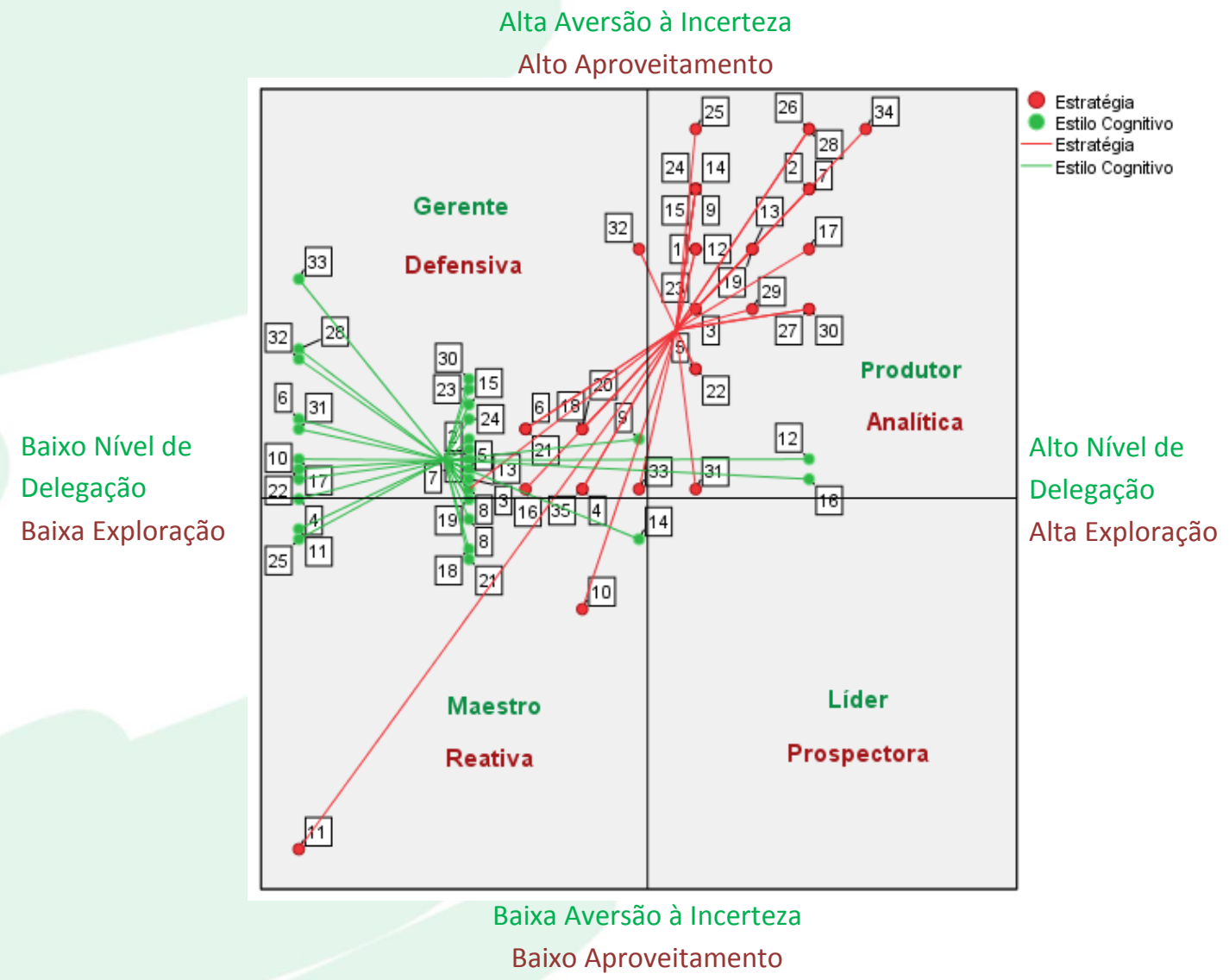

Figura 2 - Representação gráfica dos estilos cognitivos de executivo e das estratégias Fonte: Elaborado pelos autores (2012)

A análise da figura demonstra a pouca variação de estilos cognitivos dos executivos. Existe uma grande concentração no quadrante Gerente, mas mesmo os executivos que foram categorizados nos demais estilos estão muito próximo do quadrante superior direito. Por outro lado, as estratégias das empresas estão mais dispersas. Além de demonstrar a concentração do estilo cognitivo dos executivos pesquisados no quadrante Gerente e a concentração da estratégia do tipo Analítica, a figura também apresenta os alinhamentos do modelo de Håkonsson et. al. (2012) entre estratégia e estilo cognitivo. Os alinhamentos e desalinhamentos identificados estão detalhados na Tabela 2.

Tabela 2 - Detalhamento dos casos de alinhamento e desalinhamento

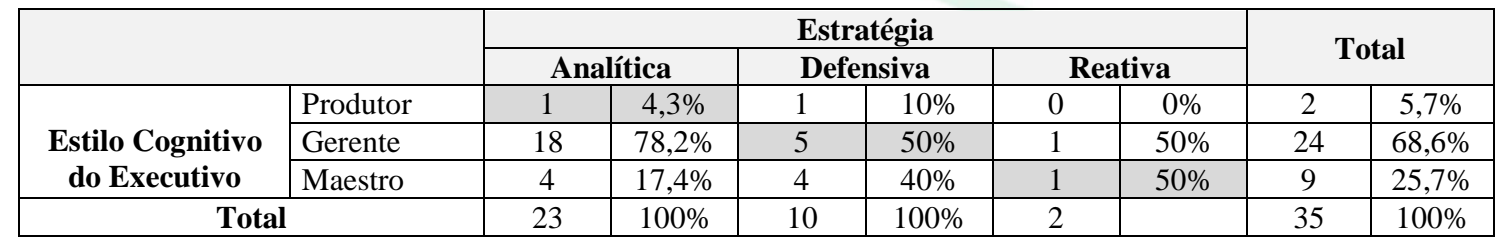

Fonte: Elaborado pelos autores (2012) 
Implementação Estratégica e Estilo do Executivo: Um Estudo sobre Alinhamento Estratégico e Desempenho em Pequenas e Médias Indústrias do Rio Grande do Norte

De acordo com os casos analisados, pode-se visualizar sete casos de alinhamento, o que representa $20 \%$ da amostra pesquisada. Um deles se situa no quadrante Maestro-Reativa; um caso está no quadrante Produtor-Analítica; e os demais casos se situam no quadrante Gerente-Defensiva. O principal desalinhamento está no par Gerente-Analítica, e o principal problema deste desalinhamento é que a estratégia Analítica, por possuir o caráter inovador oriundo da estratégia Prospectora, requer do executivo um maior grau de delegação para que este possa vislumbrar melhor as oportunidades para a empresa.

\subsection{Análise do Alinhamento e Desempenho Financeiro}

Depois de identificados os casos de alinhamento e desalinhamento entre estratégia e estilos cognitivos dos executivos, foram executados os testes de hipóteses para testar o modelo proposto. Pelo tamanho da amostra, os dados foram testados quanto à sua normalidade através do teste de Shapiro-Wilk (cf. Tabela 3).

Tabela 3 -Teste de Normalidade

\begin{tabular}{|l|c|c|c|c|}
\hline \multirow{2}{*}{ Alinhamento } & \multicolumn{3}{|c|}{ Shapiro-Wilk } \\
\cline { 3 - 5 } & & Estatística & Graus de Liberdade & Sig. \\
\hline \multirow{2}{*}{ Desempenho } & Sim &, 787 & 7 &, 030 \\
\cline { 2 - 5 } & Não &, 767 & 28 &, 000 \\
\hline
\end{tabular}

Fonte: Elaborado pelos autores (2012)

Os resultados do teste de normalidade demonstram que tanto os casos alinhados quanto os casos de desalinhamento não são normalmente distribuídos, uma vez que a significância do teste foi inferior a 0,05. Assim, para testar se há diferença estatística entre os grupos, procedeu-se o teste não paramétrico de U de Mann-Whitney para avaliar o desempenho financeiro das empresas em relação ao alinhamento entre estratégia da empresa e o estilo cognitivo do executivo. Os resultados dos testes realizados estão detalhados na Tabela 4.

Tabela 4 - Resultado do Teste de Mann-Whitney, com teste estatístico

\begin{tabular}{|c|c|c|c|c|c|}
\hline & Alinhamento & $\mathbf{N}^{\circ}$ Casos & $\begin{array}{c}\text { Classificação Média } \\
\text { do Desempenho }\end{array}$ & $\begin{array}{c}\text { Mann- } \\
\text { Whitney U }\end{array}$ & Valor $\mathbf{P}$ \\
\hline \multirow{3}{*}{ Geral (HG) } & Sim & 7 & 25,93 & \multirow{3}{*}{42,500} & \multirow{3}{*}{, $013(*)$} \\
\hline & Não & 28 & 16,02 & & \\
\hline & Total & 35 & & & \\
\hline \multirow{3}{*}{ Maestro (H1) } & Sim & 1 & 1,50 & \multirow{3}{*}{, 500} & \multirow{3}{*}{, 147} \\
\hline & Não & 8 & 5,44 & & \\
\hline & Total & 9 & & & \\
\hline \multirow{3}{*}{ Gerente (H2) } & Sim & 5 & 20,50 & \multirow{3}{*}{7,500} & \multirow{3}{*}{, $002(*)$} \\
\hline & Não & 19 & 10,39 & & \\
\hline & Total & 24 & & & \\
\hline \multirow{3}{*}{ Produtor $(\mathrm{H} 4)$} & Sim & 1 & 2,00 & \multirow{3}{*}{, 000} & \multirow{3}{*}{, 317} \\
\hline & Não & 1 & 1,00 & & \\
\hline & Total & 2 & & & \\
\hline
\end{tabular}

(*) Resultados significantes a um nível de confiança de $95 \%$

Fonte: Elaborado pelos autores (2012)

A Tabela 4 mostra a classificação média para os dois grupos testados: os casos de alinhamento entre estratégia e estilo cognitivo e os casos de desalinhamento, para todas as hipóteses da pesquisa, 
assim como os resultados dos testes estatísticos das hipóteses. Uma vez que nenhum dos executivos foi categorizado como líder, a hipótese H3 não pode ser testada.

No que se refere à hipótese geral (HG), de que o alinhamento entre o estilo cognitivo e a estratégia da empresa leva a um melhor desempenho financeiro, o resultado indica que o grupo alinhado teve maior desempenho financeiro, em relação à classificação média. $\mathrm{O}$ valor de $\mathrm{p}$ indica que esta diferença entre os desempenhos dos grupos analisados é significante a um nível de confiança de $95 \%$.

Apenas outra hipótese apresentou resultado estatisticamente significativo: a do alinhamento entre o estilo Gerente e a estratégia Defensiva (H2). O resultado do teste indica a diferença estatisticamente significativa entre o desempenho dos grupos, sendo o desempenho do grupo alinhado superior ao desempenho do grupo desalinhado.

Quanto às demais hipóteses testadas, os resultados encontrados não foram estatisticamente relevantes, pois apresentaram valor de $\mathrm{p}$ superior ao nível de significância de 5\%. É interessante ressaltar, no entanto, o resultado para as empresas que têm o executivo na categoria Maestro (H1): o desempenho financeiro das empresas cujas estratégias estão desalinhadas com este estilo do executivo foi superior ao desempenho financeiro das empresas cujas estratégias estão alinhadas, ou seja, que tem a estratégia Reativa. Este resultado indica que, embora o estilo Maestro seja inapropriado para a implementação das demais estratégias do modelo, seja pelo baixo grau de delegação, seja pelo foco restrito em ações de curto prazo; a estratégia reativa, em si, não é uma estratégia viável, que seja sustentável ao longo do tempo (MILES; SNOW, 1978; OBEL, 1993). O resultado da última hipótese, $\mathrm{H} 4$, foi coerente com o modelo de Håkonsson et. al. (2012), no entanto, a diferença entre os desempenhos não foi estatisticamente relevante.

Os resultados da presente pesquisa corroboram os achados de Håkonsson et. al. (2012). De maneira geral, os resultados da pesquisa original indicam que o alinhamento entre o estilo cognitivo do executivo e a estratégia tem impacto positivo no desempenho da empresa, e os desalinhamentos geram queda no desempenho.

\section{CONSIDERAÇÕES FINAIS}

Neste estudo, foram analisadas as pequenas e médias indústrias de transformação de Mossoró-RN, com o intuito de verificar a existência de alinhamento entre o estilo do executivo, as estratégias dessas empresas e os impactos no desempenho. Os resultados apontam que a grande maioria dos executivos das organizações estudadas se enquadrou no estilo Gerente, totalizando $68,6 \%$. No que se refere às estratégias diagnosticadas, a partir do agrupamento das variáveis operacionais, $65,7 \%$ dos respondentes categorizaram a estratégia da empresa como Analítica. Além da concentração do estilo cognitivo dos executivos pesquisados no quadrante Gerente e a concentração da estratégia do tipo Analítica, a pesquisa também apresentou os alinhamentos do modelo de Håkonsson et. al. (2012) entre estratégia e estilo cognitivo: apenas $20 \%$ das empresas pesquisadas estão alinhadas e, de maneira geral, o desempenho financeiro destas empresas foi superior ao desempenho financeiro das empresas desalinhadas.

Conclui-se que o resultado prevalecente nas empresas pesquisadas é o desalinhamento entre o estilo Gerente e a estratégia Analítica. Este grupo de empresas, no entanto, apresentou desempenho inferior ao grupo de empresas cujo estilo do executivo estava alinhado com a estratégia organizacional. A presente pesquisa não se propôs a exaurir o tema, e teve algumas limitações, tais como a dimensão e a não aleatoriedade da amostra. A tendência de melhor desempenho do estilo executivo alinhado a estratégia organizacional, merece uma análise mais aprofundada, pois, se realmente ocorre essa relação pode ser considerado um fator crítico de sucesso para as empresas. Ressalta-se ainda que os resultados não devem ser generalizados e novas pesquisas com outros setores e/ou amostras maiores sejam realizadas para fins de validação dessa conclusão.

\section{REFERÊNCIAS}

Andrews, K.R. 1996. The concept of corporate strategy. In: McKiernan, Peter (ed.). Historical Evolution of Strategic Management. Vol. I. Brookfield, Darmouth Publishing Company, p.1544.

Caetani, et al A execução estratégica na perspectiva da gestão dos processos internos: oportunidades e desafios em uma refinaria de petróleo. Encontro Anual da Associação Nacional de Pesquisa e PósGraduação em Administração, 35., 2011, Rio de Janeiro. Anais... Rio de Janeiro: Enanpad, 2011.

Cooper, A. C. Strategic Management: new ventures and small business.Long Range Planning, v. 14, n.5, p. 39-45, 1981.

Cooper, R. D., Schindler, P. S. Métodos de pesquisa em Administração. 7. ed. Porto Alegre: Bookman, 2003. 
Donato, J.V.. Fatores de sobrevivência de novas empresas. Informe ETENE- Macroeconomia, Indústria e Serviços. Banco do Nordeste, ano V, N. 4, 2011.

Fávero, L. P. et al. Análise de Dados: modelagem multivariada . Rio de Janeiro: Elsevier, 2009.

Ferreira, J. M.; Ramos, S. C. Escolha estratégica e estilos cognitivos: uma correlacao da tipologia de Miles e Snow e o modelo de Kirton. In: Encontro Nacional de Engenharia da Producao, 24., 2004, Florianopolis. Anais... Florianopolis: ABEPRO, 2004.

Gil, A. C. Como elaborar Projetos de Pesquisa. 4.ed. São Paulo: Atlas, 2002.

Gimenez, F., A. P.; Ramos, S., C.; Ferreira, J. M. O papel da análise da concorrência na formulação da estratégia em pequenas empresas. In:

Encontro de Estudos Sobre Empreendedorismo e Gestão de Pequenas Empresas (EGEPE), 4., 2005, Curitiba. Anais... Curitiba, 2005.

Gimenez, Fernando A. P. et al . Estratégia em pequenas empresas: uma aplicação do modelo de Miles e Snow. Revista de Administração Contemporânea, Curitiba, v. 3, n. 2, 1999.

Ghoshal, S., Miles, R.E., Snow, C.C., 2003. Enduring insights for managers. The Academy of Management Executive v. 17 n. 4, 109-114.

Hall, R. H. Organizações: estruturas e processos. Rio de Janeiro: Prentice-Hall do Brasil, 1984.

Hambrick, D.C. On the staying power of defenders, analyzers, and prospectors. The Academy of Management Executive. v. 17 n.4, p. 115-118, 2003.

Håkonsson, Dorthe D. et al Strategy Implementation Requires the Right Executive Style: Evidence from Danish SMEs. Long Range Planning, v.45, p. 182208, 2012.

Hrebiniak, L. G. Obstacles to effective strategy implementation. Organizational Dynamics, vol. 35, n. 1, p. 12-31, 2006.

Hitt, M. A.; Ireland, R. D.; Hoskisson, R.. Strategic management. New York: South-Western Pub, 2008.

Jansen, J.J.P., et.al. Senior team attributes and organizational ambidexterity: The moderating role of transformational leadership. Journal of Management Studies v. 45, n. 5, p. 982-1007, 2008.

Kaplan, R.S.; Norton D.P. The balanced scorecard: translating strategy into action. Boston: Harvard Business School Press, 1996.

Kerlinger, F. N. A metodologia da pesquisa em ciências sociais: um tratamento conceitual. São Paulo: EPU, 1980.

Kirton, M.; Bailley, A.; Glendinning, W. Adaptors and innovators:preference for educational studies procedures. Journal of Psychology, v. 125, n. 4, p. 445-456, 1991.

Korunka, C. et al. The entrepreneurial personality in the context of resources, environment, and the startup process - a configurational approach. Entrepreneurship theory and practice, p. 23-42, 2003.

Lima, E. Aprendizagem e difusão de inovações tecnológicas entre micro, pequenas e médias empresas: o caso de WK Sistemas, de Blumenau. In: Encontro de Estudos sobre Emprendedorismo e Gestão de Pequenas Empresas, 3., 2003, Brasilia. Anais...Brasília: [s.n.], 2003.

Miles, R. E; Snow, C. C. Organizational strategy, structure and process. New York: McGraw-Hill, 1978.

Mintzberg, H., \& Waters, J.A. 1996. Of Strategies, Deliberate and Emergent.In: McKiernan, Peter (ed.). Historical Evolution of Strategic Management.v. 2.Brookfield, Darmouth Publishing Company, p. 413-428.

Mintzberg, Henry; Quinn, James, B. O processo da estratégia. 3. ed. Porto Alegre: Bookman, 2001.

Mintzberg, H.; Ahlstrand; B. Lampel, J. Safári de Estratégia: um roteiro pela selva do planejamento estratégico.2.ed. Porto Alegre: Bookman, 2005.

Najberg, S.; Puga, F. P.; Oliveira, P. A. S. Criação e fechamento de firmas no Brasil: dezembro de 1995 / dezembro 1997. Rio de Janeiro: BNDES: Textos para discussão, n $^{\circ}$ 79, 2000.

Nobre, L. H. N. Estrutura de capital e níveis de endividamento da PME's cearenses. $160 \mathrm{f}$. Dissertação (Mestrado em Administração) - Curso de Mestrado Acadêmico em Administração da 

em Pequenas e Médias Indústrias do Rio Grande do Norte

Universidade Estadual do Ceará. Fortaleza: UECE, 2005.

Obel, B., Strategi og LedelseeEr der en sammenhæng? Strategi og Ledelse. Veje og Visioner mod_ar 2000, p. 396-408. 1993.

Porter, M. Estratégia Competitiva: técnicas para análise de indústrias e da concorrência.7.ed. Rio de Janeiro: Campus, 1986.

Porter, M. E. A nova era da estratégia. HSM management Edição especial, março - abril, 2000.

Ramos, S. C. Isomorfismo mimético e contexto de referência: um estudo em pequenas empresas de
Curitiba/PR. 2005. 49f. Dissertação (Mestrado em Administração) - Pontifícia Universidade Católica do Paraná, 2005.

Rocha, L. M. A teoria financeira no contexto das pequenas e médias empresas:o caso do sector têxtil e de vestuário em Portugal. Lisboa: Sociedade Gestora de Mercados Regulamentados, série: Moderna Finança, n 24, 2001.

Santos, L. L. S. ; Alves, R.C.; Almeida, K. N. T.. Formação de estratégia nas micro e pequenas empresas: um estudo no centro-oeste mineiro. RAE - Revista de Administração de Empresas, vol. 47, 2007. 